\title{
A case of severe open bite malocclusion with crowding and short roots treated by two-jaw surgery including four-piece LeFort I osteotomy
}

\section{Mitsuhiro Hoshijima}

Okayama University: Okayama Daigaku

Naoki Oka

Okayama University: Okayama Daigaku

\section{Tatsushi Matsumura}

Okayama University: Okayama Daigaku

\section{Seiji lida}

Okayama University: Okayama Daigaku

Hiroshi Kamioka ( $\nabla$ kamioka@md.okayama-u.ac.jp )

Okayama Univ.

\section{Case report}

Keywords: anterior open bite, short roots, severe crowding, four-piece segmental LeFort I osteotomy

Posted Date: July 19th, 2021

DOl: https://doi.org/10.21203/rs.3.rs-699570/v1

License: (9) This work is licensed under a Creative Commons Attribution 4.0 International License. Read Full License 


\section{Abstract}

\section{Background}

Appropriate operations in severe anterior open bite (AOB) cases are extremely complicated to perform because of the multiple surgical procedures involved, difficulty of predicting posttreatment aesthetics and high relapse rate.

\section{Case report}

We herein report a 16-year-old girl with skeletal Class II, severe AOB malocclusion and crowding with short roots and aesthetic and functional problems. Four-piece segmental LeFort I osteotomy combined with a posterior horseshoe-like osteotomy was performed for maxillary intrusion, and sagittal split ramus osteotomy (SSRO) and genioplasty were performed for mandibular advancement. The malocclusion and skeletal deformity were significantly improved by the surgical orthodontic treatment. Functional and aesthetic occlusion with an improved facial profile was established, and no further root shortening was observed. Acceptable occlusion and dentition were maintained after a two-year retention period.

\section{Conclusion}

This strategy of surgical orthodontic treatment with a complicated operative procedure might be effective for managing severe AOB malocclusion.

\section{Background}

Anterior open bite (AOB) includes various etiologies, such as an abnormal tongue, pernicious habits, respiration issues, neurological disturbances and muscular dystrophy, and the dental and skeletal morphology of patients varies markedly [1-3]. Because of the multiple genetic and environmental factors involved, nongrowing patients with AOB are difficult to treat with orthodontics [4]. Treatment options for patients with $A O B$ include the use of elastics combined with the multiloop edgewise archwire technique [5]. The use of titanium miniscrews as orthodontic anchorage devices also makes the counterclockwise rotation of the mandible possible because of the intrusion of the molars [6, 7].

The general treatment for adult patients with a skeletal AOB has been a combination of orthodontic therapy and orthognathic surgery [4]. In patients with severe skeletal AOB, more complicated operations, such segmental maxillary osteotomy and differential maxillary impaction, may be required, including horseshoe palatal osteotomy in combination with LeFort I osteotomy [8]. Therefore, AOB treatment is considered strategic because of difficulties in performing manifold operative procedures and the potential for relapse $[9,10]$. Furthermore, patients with $A O B$ can have a shortened root length and high incidence of root resorption with orthodontic treatment [11]. This is one reason for the difficulty in treating $A O B$ patients. 
We herein report the efficiency of minimum tooth movement for short roots and an effective strategy using optimal two-jaw surgery to correct a severe skeletal AOB deformity.

\section{Case Presentation}

\section{Diagnosis and Etiology}

A 16-year-old girl presented at the outpatient department of our hospital with a chief complaint of an inability to bite with her front teeth. Given the lack of contact of the anterior teeth with each other, she was deemed to have articulation impairment and masticatory disturbance. Facial photographs showed a symmetrical face, a convex profile and a long lower facial height. She also had an obtuse nasolabial angle and no lip seal (Fig. 1A).

The mandibular dental midline was deviated $1.5 \mathrm{~mm}$ toward the right compared with the maxilla, and the occlusal plane was canted (Fig. 1A). Although the stability of the mandibular position in occlusion was slightly flexible, a Class I relationship was observed at the centric occlusion bilaterally. Her overjet was 9.0 $\mathrm{mm}$, and her anterior open bite was $9.5 \mathrm{~mm}$. There was severe crowding in the maxillary and mandibular arch, and their arch length discrepancies were -10.7 and $-12.6 \mathrm{~mm}$, respectively (Fig. 1B). The cephalometric analysis showed a skeletal Class II jaw-base relationship, a high mandibular plane angle, normal maxillary incisor positioning and proclined mandibular incisors (Fig. 2A and Table 1) [12]. The posteroanterior cephalogram showed that the center of the maxillary and mandibular arch accorded with the facial midline (Fig. 2B). The panoramic and periapical radiographs showed a shortened root for all incisors and canines (Fig. $2 \mathrm{C}$ and D). In addition, a panoramic radiograph showed that all third molars were impacted (Fig. 2C). A six-degrees-of-freedom jaw movement recording system showed that shortrange movements of the incisal path were observed during maximum open-close jaw movements because of her severe open bite (Fig. 3A). 
Table 1

Cephalometric summary.

\begin{tabular}{|cccccc|}
\hline & \multicolumn{2}{l}{ Japanese norm (adult) } & Pretreatment & Posttreatment & Postretention \\
\cline { 2 - 3 } & Mean & SD & & & \\
\hline Angular $\left(^{\circ}\right)$ & & & & & \\
\hline ANB & 2.8 & 2.4 & 7.5 & 5.0 & 5.5 \\
\hline SNA & 80.8 & 3.6 & 73.5 & 74.5 & 74.5 \\
\hline SNB & 77.9 & 4.5 & 66.0 & 69.5 & 69.0 \\
\hline U1-FH & 112.3 & 8.3 & 113.5 & 110.0 & 110.0 \\
\hline L1-FH & 56.0 & 8.1 & 35.5 & 53.0 & 52.5 \\
\hline L1-Mp & 93.4 & 6.8 & 87.5 & 80.5 & 80.5 \\
\hline Mp-FH (FMA) & 30.5 & 3.6 & 57.0 & 46.5 & 47.0 \\
\hline Linear (mm) & & & & & \\
\hline Overjet & 3.1 & 1.1 & 9.0 & 3.0 & 2.5 \\
\hline Overbite & 3.3 & 1.9 & -9.5 & 1.5 & 0.5 \\
\hline Ar-Go & 47.3 & 3.3 & 71.0 & 77.5 & 77.5 \\
\hline Ar-Me & 106.6 & 5.7 & 112.0 & 113.5 & 113.5 \\
\hline
\end{tabular}

\section{Treatment objectives and alternative options}

Based on these findings, the patient was diagnosed with skeletal open bite malocclusion, a skeletal Class II jaw-base relationship and severe crowding with shortened roots.

Since the treatment objectives were to correct the skeletal deformity causing open bite and obtain ideal occlusion and the patient was already fully grown, the ideal treatment was a combination of surgery and orthodontic therapy. Four-piece LeFort I maxillary advancement osteotomy with differential impaction of the posterior segment and positioning of the anterior segment were planned to correct the open bite and maxillary incisor display. After autorotation of the mandible, bilateral sagittal split osteotomy and genioplasty could be considered to correct the open bite and long face. All impacted third molars were to be extracted before surgery so as not to interfere with the surgical procedures.

Another option was camouflage orthodontic treatment using miniscrew anchorage for the intruding maxillary and mandibular molars. An improvement of the anterior open bite could thus be obtained by the autorotation of the mandible. However, this approach would not be able to sufficiently improve the open bite and long face, which were severe skeletal deformities. Furthermore, it was feared that the shortened root of the incisors might be exacerbated by excessive tooth movement. To manage these issues, we 
decided to perform a combination of surgery and orthodontic therapy to improve both the dentofacial morphology and stomatognathic functions.

\section{Treatment progress}

After extraction of all canines, $0.018 \times 0.025$-inch preadjusted edgewise appliances were bonded to both arches, except for the maxillary incisors. Leveling and alignment were started with 0.016 -inch heatactivated nickel-titanium wires for the maxilla and mandible, respectively. After performing leveling and alignment for 7 months, 0.018-inch preadjusted edgewise appliances were bonded to the maxillary incisors. The initial alignment was achieved with 0.016-inch heat-activated nickel-titanium wires. Subsequently, tooth alignment was performed by changing the archwires sequentially, and $0.016 \times 0.022-$ inch stainless steel wires were used to adjust the tooth positions before surgery. The mandibular canines were extracted in order to improve the severe crowding and the missing canines were substituted with first premolars (Fig. 4). All third molars were extracted during leveling and alignment six months before surgery.

After presurgical orthodontic treatment for 1 year 10 months, 4-piece segmental LeFort I osteotomy combined with posterior horseshoe-like osteotomy was performed (Fig. 5). Maxillary osteotomy was achieved with posterior intrusion of $4.5 \mathrm{~mm}$ and advancement of $2.0 \mathrm{~mm}$ at the level of the first molars. Sagittal split ramus osteotomy (SSRO) of the mandible was performed with counterclockwise rotation and advancement of $10.0 \mathrm{~mm}$ at B point. Genioplasty was performed with advancement and intrusion of $4.0 \mathrm{~mm}$ each. Following osteotomy, the maxillary anterior segment was repositioned practically unchanged and connected to the mandible with appropriate overjet.

In the postsurgical orthodontic treatment, $0.016 \times 0.022$-inch stainless steel wires were installed to induce space closure of the maxillary dental arch spaces. Postsurgical orthodontic treatment was performed for 9 months. Detailing was initiated with $0.016 \times 0.022$-inch stainless steel wires in both arches, and then the edgewise appliances were removed. The total active treatment period was 2 years 11 months. The maxillary and mandibular wrap-around retainers were placed, and the patient was followed for two years.

\section{Treatment results}

The treatment induced impaction of the maxillary posterior pieces and the counterclockwise rotation of the mandibular segment, which subsequently improved the patient's facial profile and incompetent lip seal (Fig. 6A). The AOB and severe crowding were improved, and an adequate overjet and overbite were achieved. Class I molar relationships were obtained (Fig. 6B).

On an evaluation of the jaw movements after treatment using a jaw-movement recording system with sixdegrees-of-freedom, increases in the range of the maximum open-close jaw movements were observed. Furthermore, the condylar movement was maintained on both sides after orthodontic treatment (Fig. 3B).

The posttreatment cephalometric evaluation showed a decrease in the ANB angle, and a skeletal Class I jaw relationship was achieved (Fig. 7A, Table 1). The surgical changes in hard tissue resulted in maxillary 
advancement, posterior impaction and anterior segment repositioning as well as the subsequent mandibular counterclockwise rotation. The posterior segment of the maxilla eventually demonstrated intrusion of $4.5 \mathrm{~mm}$ and advancement of $2.0 \mathrm{~mm}$.

The mandible was also set forward about $15 \mathrm{~mm}$ at the pogonion by SSRO and genioplasty. A posteroanterior cephalogram showed that the maxillary and mandibular midline coincided with the facial midline (Fig. 7B). A significant change in the maxillary incisor inclination was not shown, indicating that the relevant incisor inclination had been maintained (Fig. 8, Table 1). Suitable root paralleling was observed on a panoramic radiograph (Fig. $7 \mathrm{C}$ ). The posttreatment panoramic and periapical radiographs showed no remarkable apical root resorption or alveolar bone loss (Fig. 7C, D).

The duration of active orthodontic treatment was 2 years 11 months. After two years of retention, although the cephalometric analysis showed a slight extrusion of the mandibular molars and clockwise rotation of the mandible, an acceptable facial profile and occlusion were maintained (Fig. 9-11, Table 1). The patient was satisfied with the treatment results.

\section{Discussion}

Open bite malocclusion with an interincisal distance of more than $5 \mathrm{~mm}$ is extremely difficult to treat with orthodontics alone [13]. The relatively unstable results achieved with orthodontic treatment alone make orthognathic surgery generally preferred when there are skeletal discrepancies involved. Correction of severe skeletal $A O B$ with an abnormal maxillomandibular relationship has been shown to be stable with treatment by two-jaw surgery rather than mandibular surgery alone [14]. LeFort I osteotomy with posterior impaction with mandibular osteotomy has also usually been used to treat adults with skeletal AOB [15].

In this case, since the patient had extreme skeletal AOB with a long lower facial height, including severe crowding and significant shortening of all incisor and canine roots, adequate impaction of the posterior teeth and minimum teeth movement were the main focuses of the treatment plan. Therefore, the maxillary osteotomy in this present case was performed employing four-piece LeFort I osteotomy with differential impaction and advancement of the posterior teeth segment combined with clockwise rotation of the anterior segment. The anterior-superior mandibular repositioning with counterclockwise rotation by bilateral SSRO and reduction genioplasty were useful in improving the AOB and convex profile. Furthermore, the extraction of both maxillomandibular canines in this case minimized the anterior teeth movement, which was useful given the high incidence of root resorption in patients with AOB [11]. In fact, there was no further shortening after the orthodontic treatment for about three years.

In the present treatment plan, an extremely large amount of superior and medial movement was required in the maxillary posterior segment. However, achieving this by standard LeFort I osteotomy was technically difficult, so we performed four-piece segmental LeFort I combined with posterior horseshoelike osteotomy. The strategic advantage of this technique is that the differential movement of each segment is useful for avoiding osseous interference in the perpendicular portioning of the palatal bone and the horizontal sectioning of the pterygoid plate. Such a procedure reduces the bone trimming 
required for the anterior-superior movement in the posterior segment of the maxilla and it also minimizes the risk of damaging the neurovascular bundle and palatal mucosa $[8,16]$.

In addition, the range of the incisal path during maximum mouth opening and closing was increased after orthodontic treatment, and the motion of both the incisors and the condyles was kept stable according to a gnathological analysis. These results may be attributed to the adequate improvement of the open bite, as functional occlusion was deemed to have been achieved. This surgical approach may be an efficient alternative for improving the oral aesthetics and promoting a good quality of life [17].

Four-piece segmental LeFort I combined with posterior horseshoe-like osteotomy is regarded as a useful and safe technique for resolving impaction and advancement of the maxillary posterior area. The present report indicates that orthodontic surgery featuring the combination of LeFort I osteotomy and bilateral SSRO with genioplasty helps improve the occlusion, aesthetics and stomatognathic function of severe AOB patients.

\section{Conclusion}

A case of severe open bite malocclusion with shortened roots and crowding was treated by orthognathic surgery featuring the combination of four-piece segmental LeFort I osteotomy and posterior horseshoelike osteotomy, SSRO and genioplasty. Functional and aesthetic occlusion and a generalized facial profile were established, and no further root shortening was observed after orthodontic treatment. Acceptable stability was observed after two years of retention.

\section{Declarations}

\section{Acknowledgements}

Authors would like to thank Takashi Yamashiro from Department of Orthodontics and

Dentofacial Orthopedics, Osaka University Graduate School of Dentistry, who provided valuable advice for this case report.

\section{Ethical Approval and Consent to participate}

We explained the aims and alternatives of the treatment to the patient and obtained her informed consent.

\section{Consent for publication}

The patient provided her informed consent to publish this case report.

\section{Availability of supporting data}

Not applicable. 


\section{Competing interests}

The authors declare that they have no competing interests.

\section{Funding}

Not applicable.

\section{Authors' contributions}

$\mathrm{MH}$ performed the orthodontic treatment of this case, analyzed the records, reviewed all data and wrote the case report. NO participated in the orthodontic treatment of this case. TM and SI performed the surgical procedures of this case. HK designed the case report and approved the final manuscript. All authors read and approved the final manuscript.

\section{References}

1. Rijpstra C, Lisson JA. Etiology of anterior open bite: a review. J Orofac Orthop. 2016;77:281-6.

2. Cangialosi TJ. Skeletal morphologic features of anterior open bite. Am J Orthod. 1984;85:28-36.

3. Harvold EP, Tomer BS, Vargervik K, Chierici G. Primate experiments on oral respiration. Am J Orthod. 1981;79:359-72.

4. Reichert I, Figel P, Winchester L. Orthodontic treatment of anterior open bite: a review article-is surgery always necessary? Oral Maxillofac Surg. 2014;18:271-7.

5. Kim YH, Han UK, Lim DD, Serraon ML. Stability of anterior openbite correction with multiloop edgewise archwire therapy: a cephalometric follow-up study. Am J Orthod Dentofacial Orthop. 2000;118:43-54.

6. Kuroda S, Katayama A, Takano-Yamamoto T. Severe anterior open-bite case treated using titanium screw anchorage. Angle Orthod. 2004;74:558-67.

7. Deguchi T, Kurosaka H, Oikawa H, Kuroda S, Takahashi I, Yamashiro T, Takano-Yamamoto T. Comparison of orthodontic treatment outcomes in adults with skeletal open bite between conventional edgewise treatment and implant-anchored orthodontics. Am J Orthod Dentofacial Orthop. 2011;139:60-8.

8. Shimo T, Nishiyama A, Jinno T, Sasaki A. Severe gummy smile with class II malocclusion treated with LeFort I osteotomy combined with horseshoe osteotomy and intraoral vertical ramus osteotomy. Acta Med Okayama. 2013;67:55-60.

9. Burford D, Noar JH. The causes, diagnosis and treatment of anterior open bite. Dent Update. 2003;30:235-41.

10. Epker BN, Fish L. Surgical-orthodontic correction of open-bite deformity. Am J Orthod. 1977;71:27899. 
11. Harris EF, Butler ML. Patterns of incisor root resorption before and after orthodontic correction in cases with anterior open bites. Am J Orthod Dentofacial Orthop. 1992;101:112-9.

12. Wada K, Matsushita K, Shimazaki S, Miwa Y, Hasuike Y, Susami R. An evaluation of a new case analysis of a lateral cphalometric roentgenogram. J Kanazawa Med Univ. 1981;6:60-70.

13. Kuroda S, Sakai Y, Tamamura N, Deguchi T, Takano-Yamamoto T. Treatment of severe anterior open bite with skeletal anchorage in adults: comparison with orthognathic surgery outcomes. Am J Orthod Dentofacial Orthop. 2007;132:599-605.

14. Proffit WR, Bailey LJ, Phillips C, Turvey TA. Long-term stability of surgical open-bite correction by Le Fort I osteotomy. Angle Orthod. 2000;70:112-7.

15. Park JH, Papademetriou M, Gardiner C, Grubb J. Anterior open bite correction with 2-jaw orthognathic surgery. Am J Orthod Dentofacial Orthop. 2019;155:108-16.e2.

16. Shimazaki K, Otsubo K, Yonemitsu I, Kimizuka S, Omura S, Ono T. Severe unilateral scissor bite and bimaxillary protrusion treated by horseshoe Le Fort I osteotomy combined with mid-alveolar osteotomy. Angle Orthod. 2014;84:374-9.

17. Palomares NB, Celeste RK, Miguel JA. Impact of orthosurgical treatment phases on oral healthrelated quality of life. Am J Orthod Dentofacial Orthop. 2016;149:171-81.

\section{Figures}



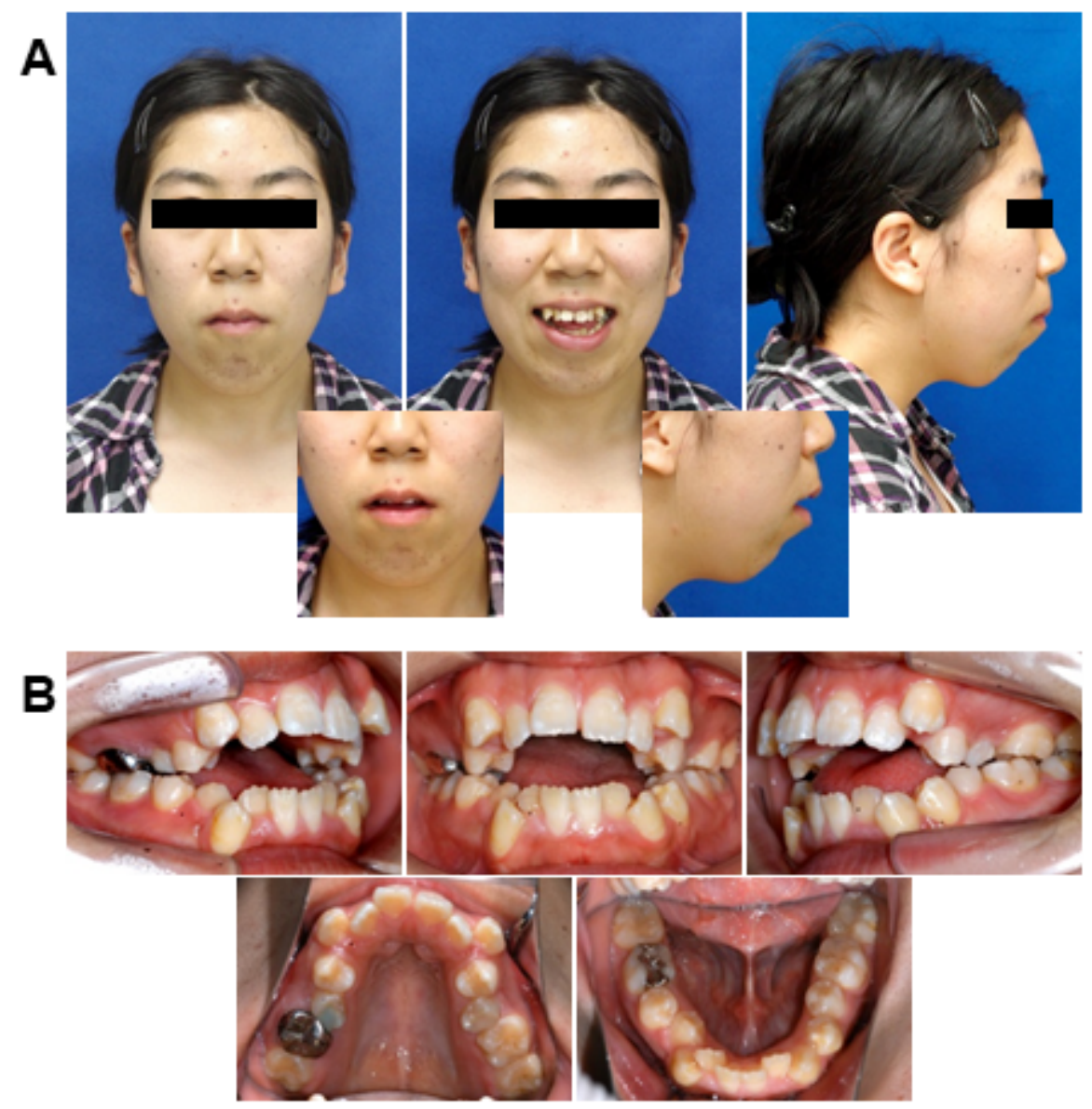

Figure 1

Pre-treatment views. A, Facial photographs, Insets: Rest position. B, Intraoral photographs. 

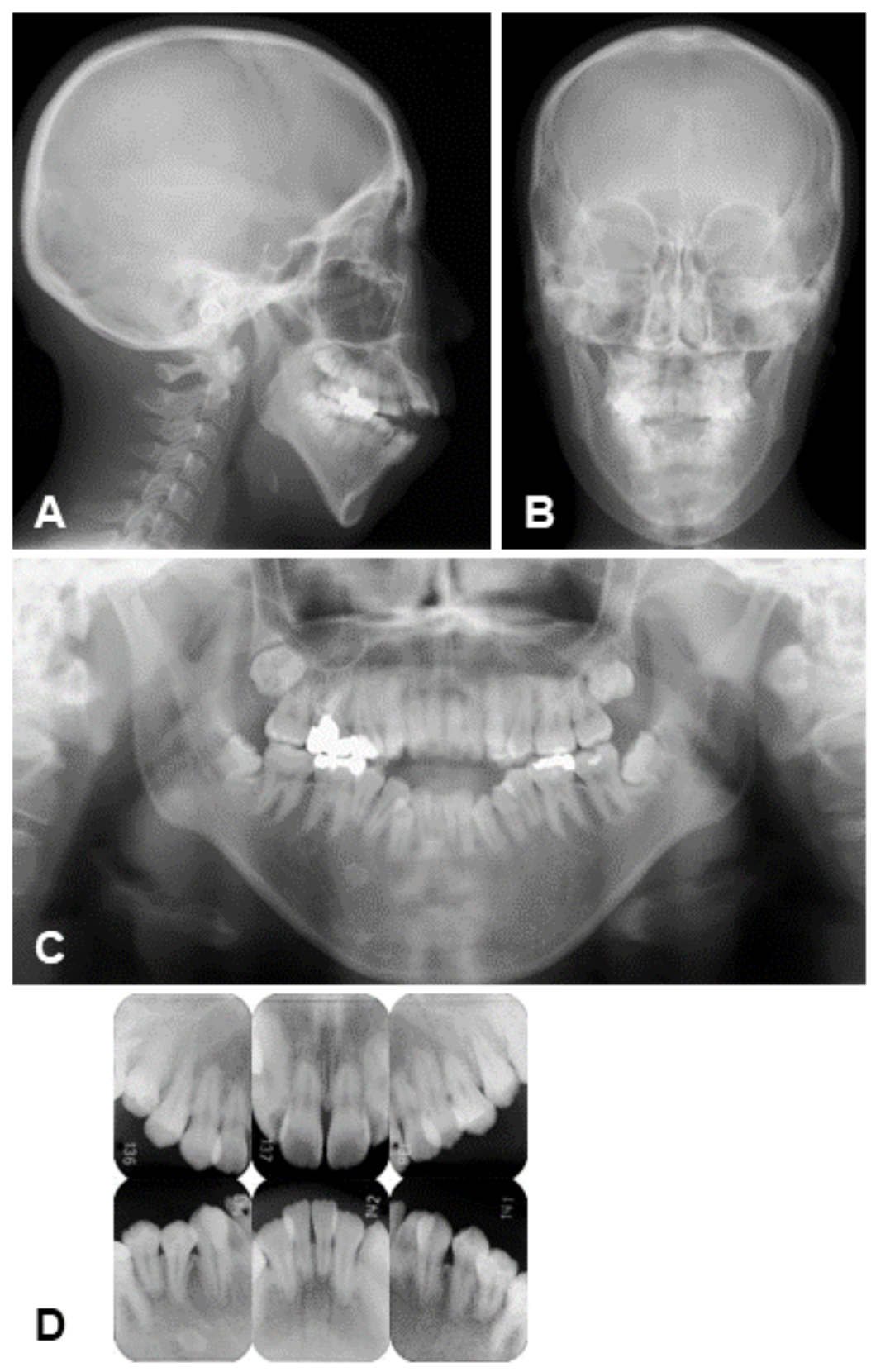

Figure 2

Pre-treatment radiographs. A, Lateral cephalogram. B, Posteroanterior cephalogram. C, Panoramic radiograph. D, Periapical radiographs. 


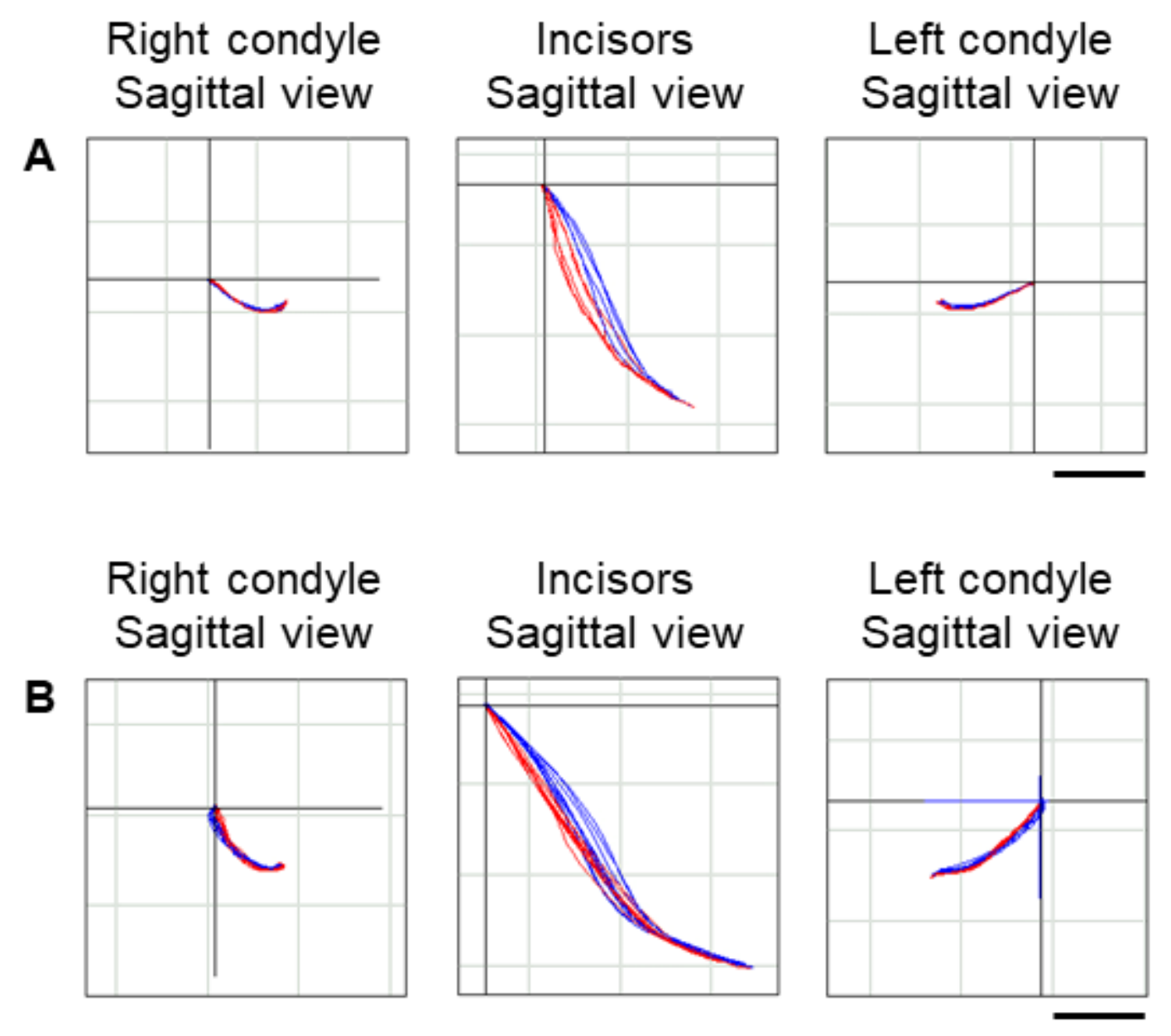

Figure 3

Condylar movements and the incisal paths, as detected using a six-degrees-of-freedom jaw movement recording system. The red line indicates the opening phase, and the blue line indicates the closing phase in maximum open-close. A, Pre-treatment. B, Post-treatment. 


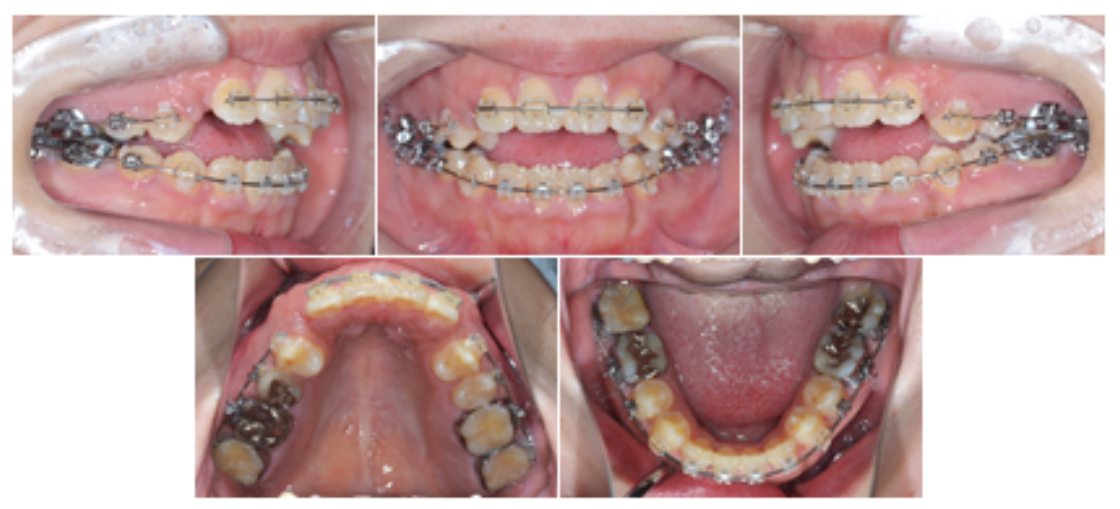

\section{Figure 4}

The treatment progress during presurgical orthodontic treatment.

A

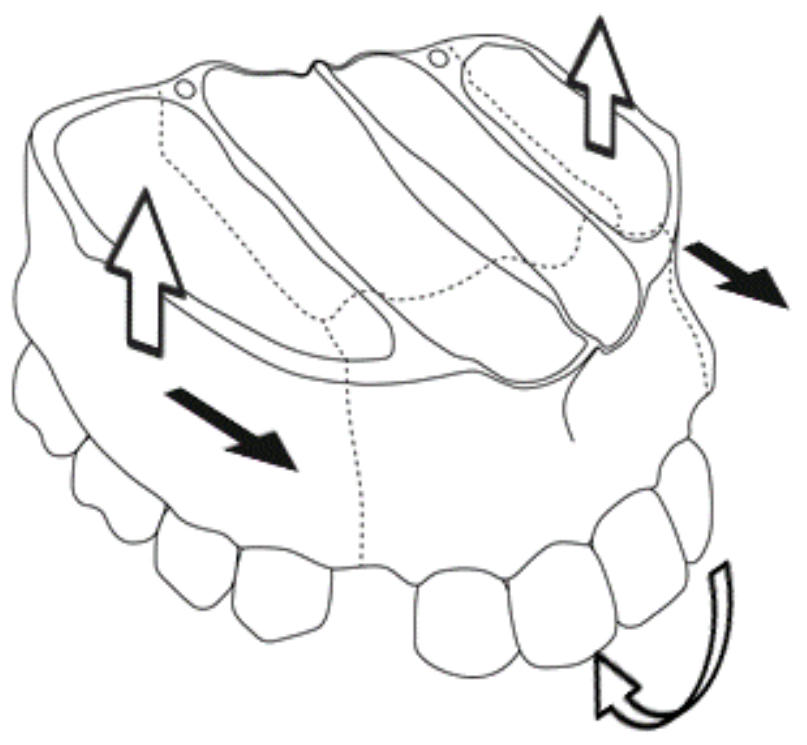

B

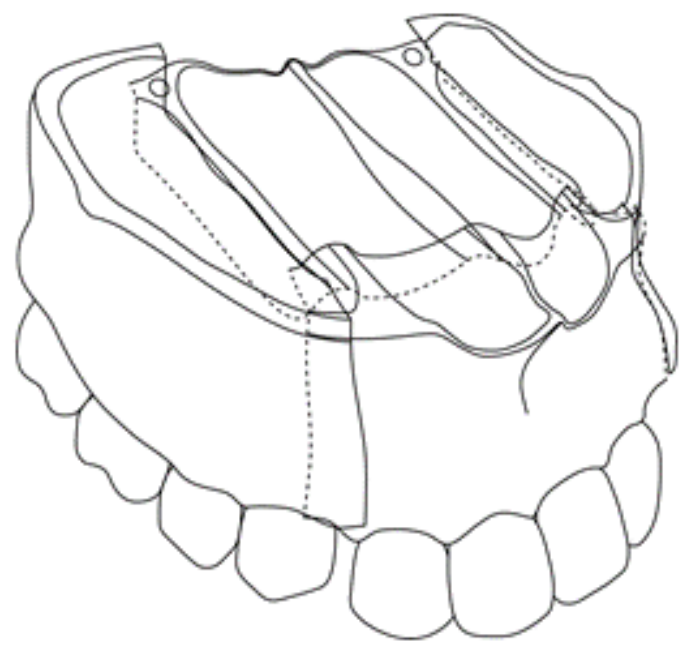

Figure 5

A schematic illustration of the four-piece segmental LeFort I osteotomy procedure. A, Before osteotomy. These segments are allowed to move, as shown by arrows. B, After osteotomy. 


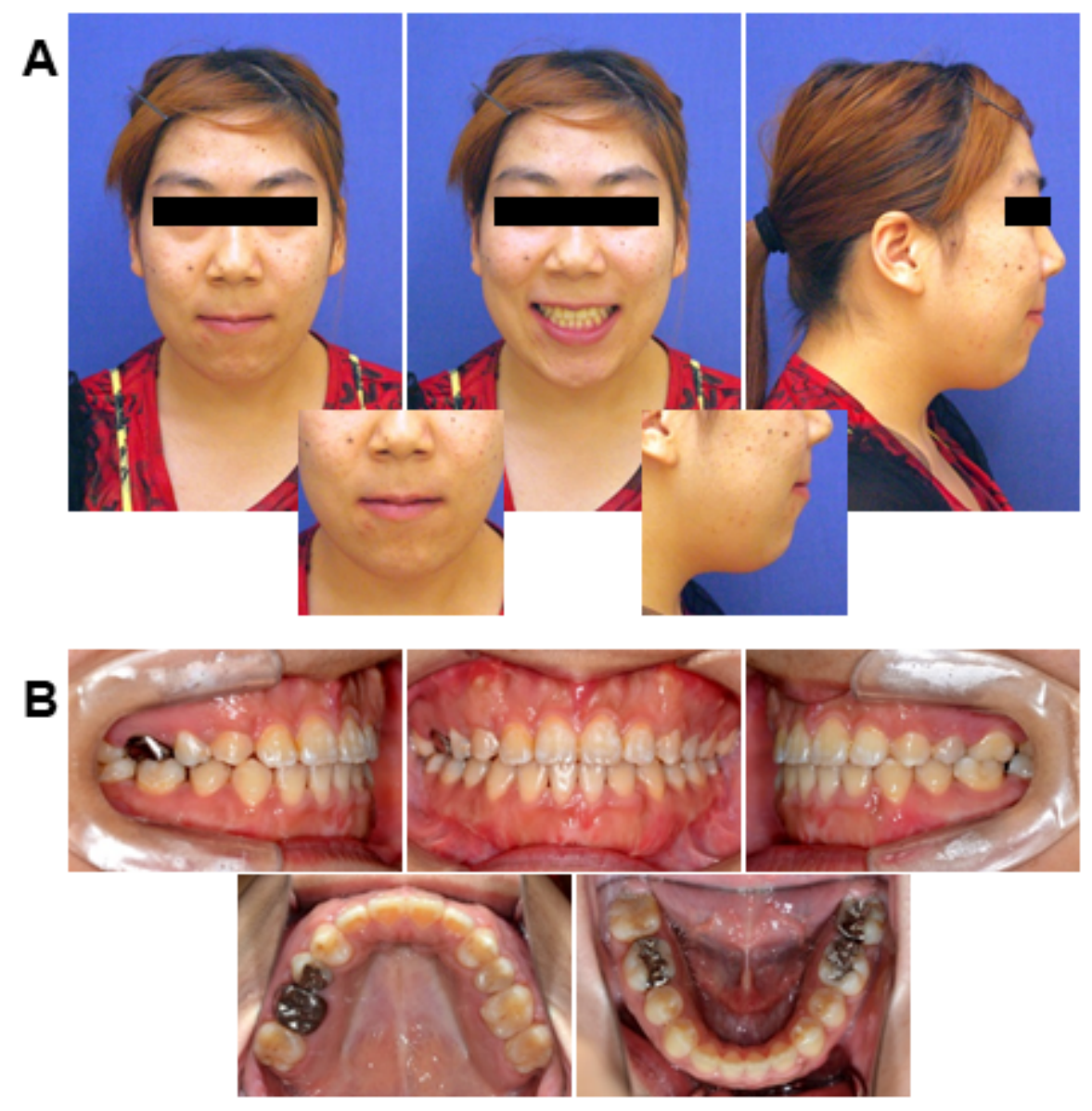

Figure 6

Post-treatment views. A, Facial photographs, Insets: Rest position. B, Intraoral photographs. 

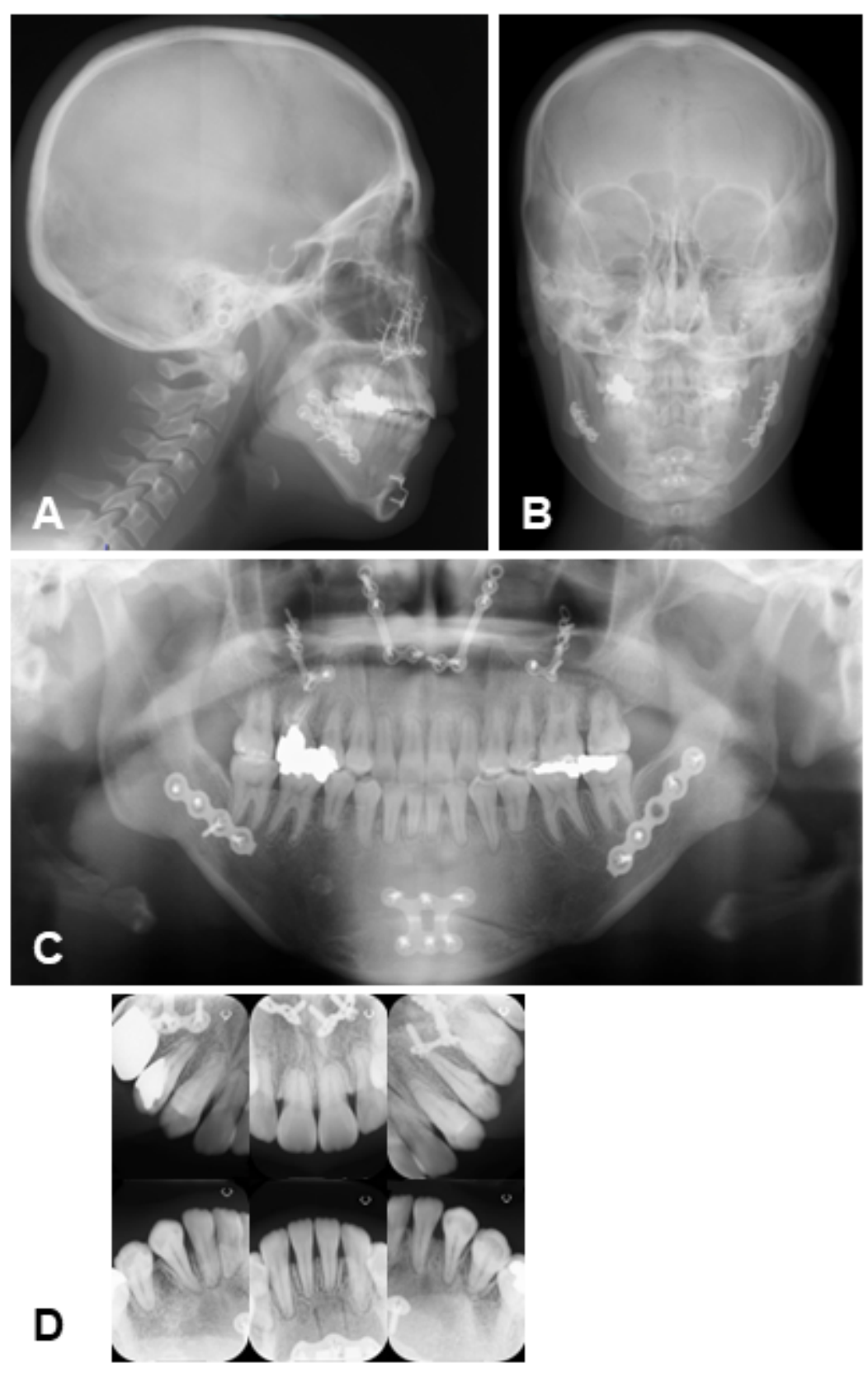

Figure 7

Post-treatment radiographs. A, Lateral cephalogram. B, Posteroanterior cephalogram. C, Panoramic radiograph. D, Periapical radiographs. 


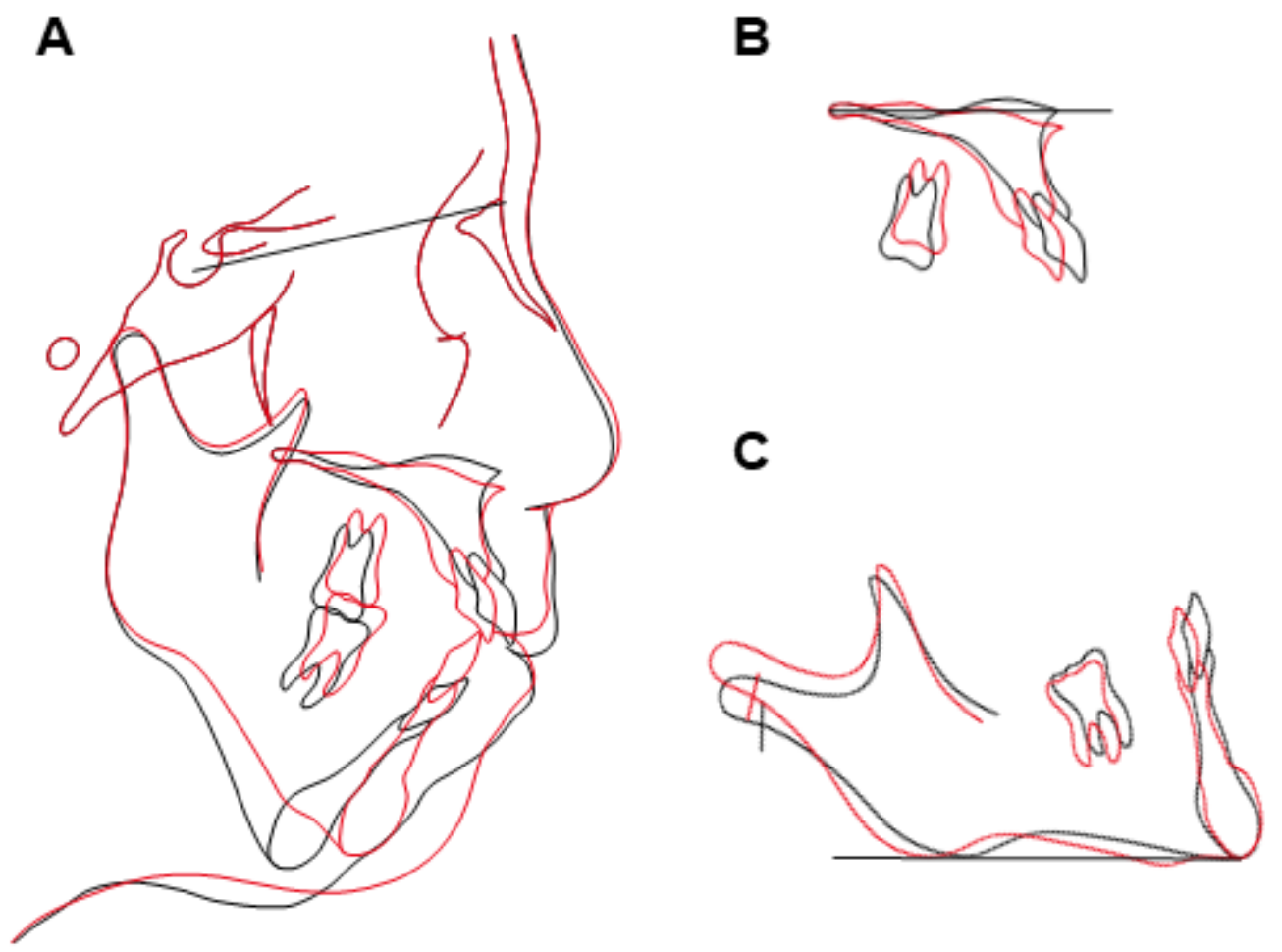

Figure 8

Superimposed cephalometric tracings show the changes from the pre-treatment (black line) to posttreatment (red line) stages. A, The overall superimposition on the sella-nasion plane at the sella. B, The superimposition on the initial palatal plane at the PNS. C, The superimposition on the mandibular plane at the menton. 

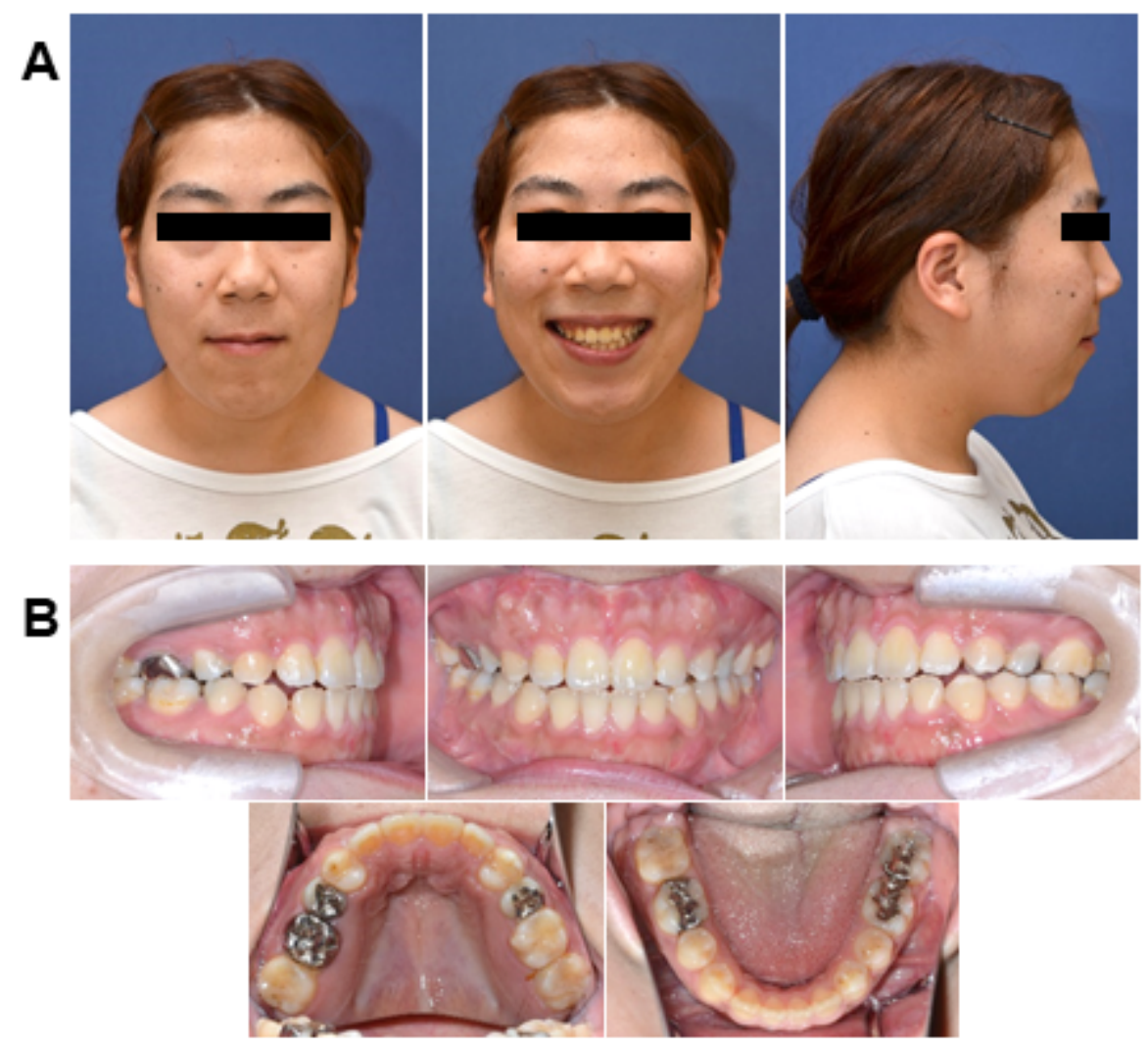

Figure 9

Post-retention views. A, Facial photographs. B, Intraoral photographs. 

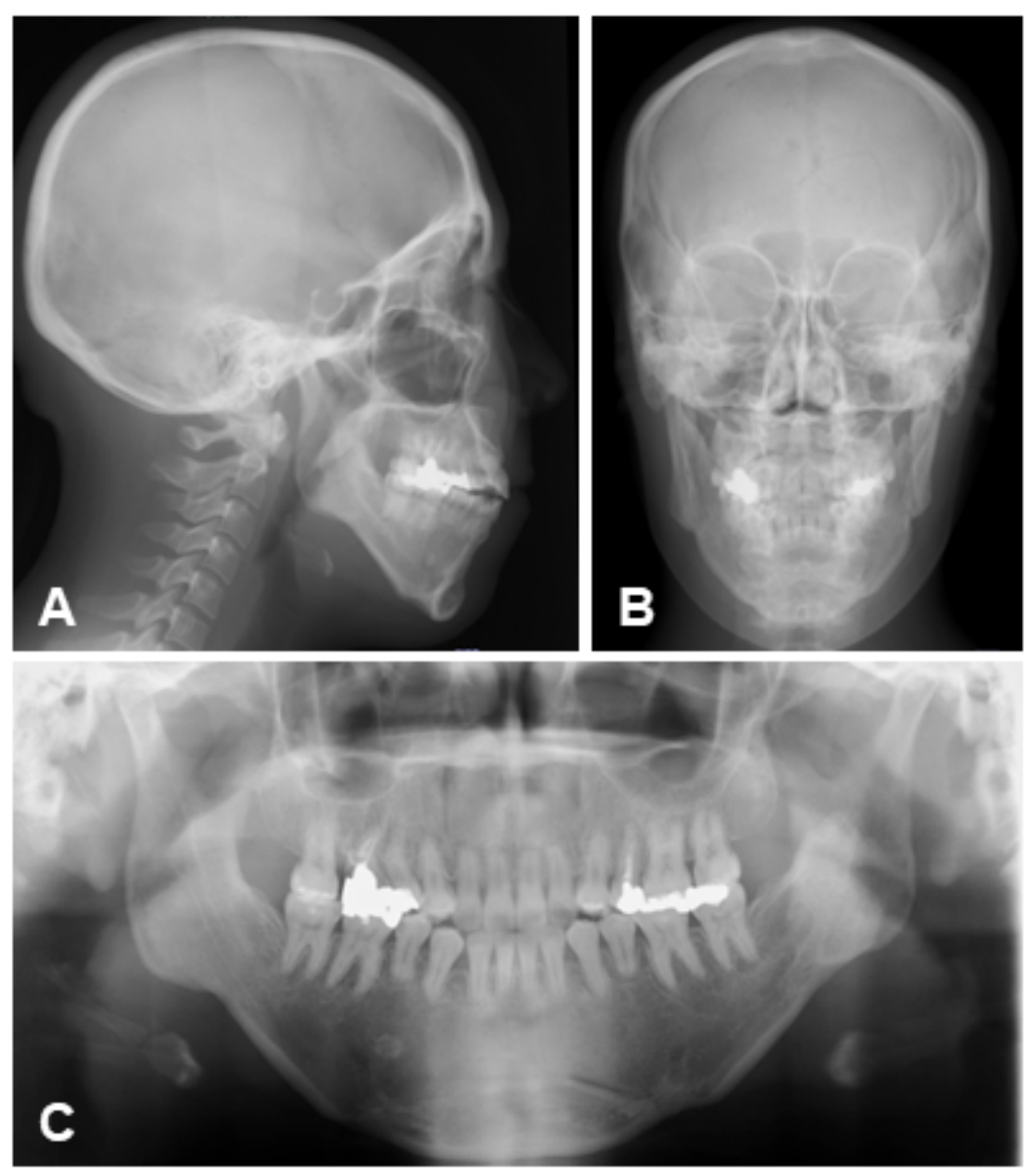

Figure 10

Post-retention radiographs. A, Lateral cephalogram. B, Posteroanterior cephalogram. C, Panoramic radiograph. 


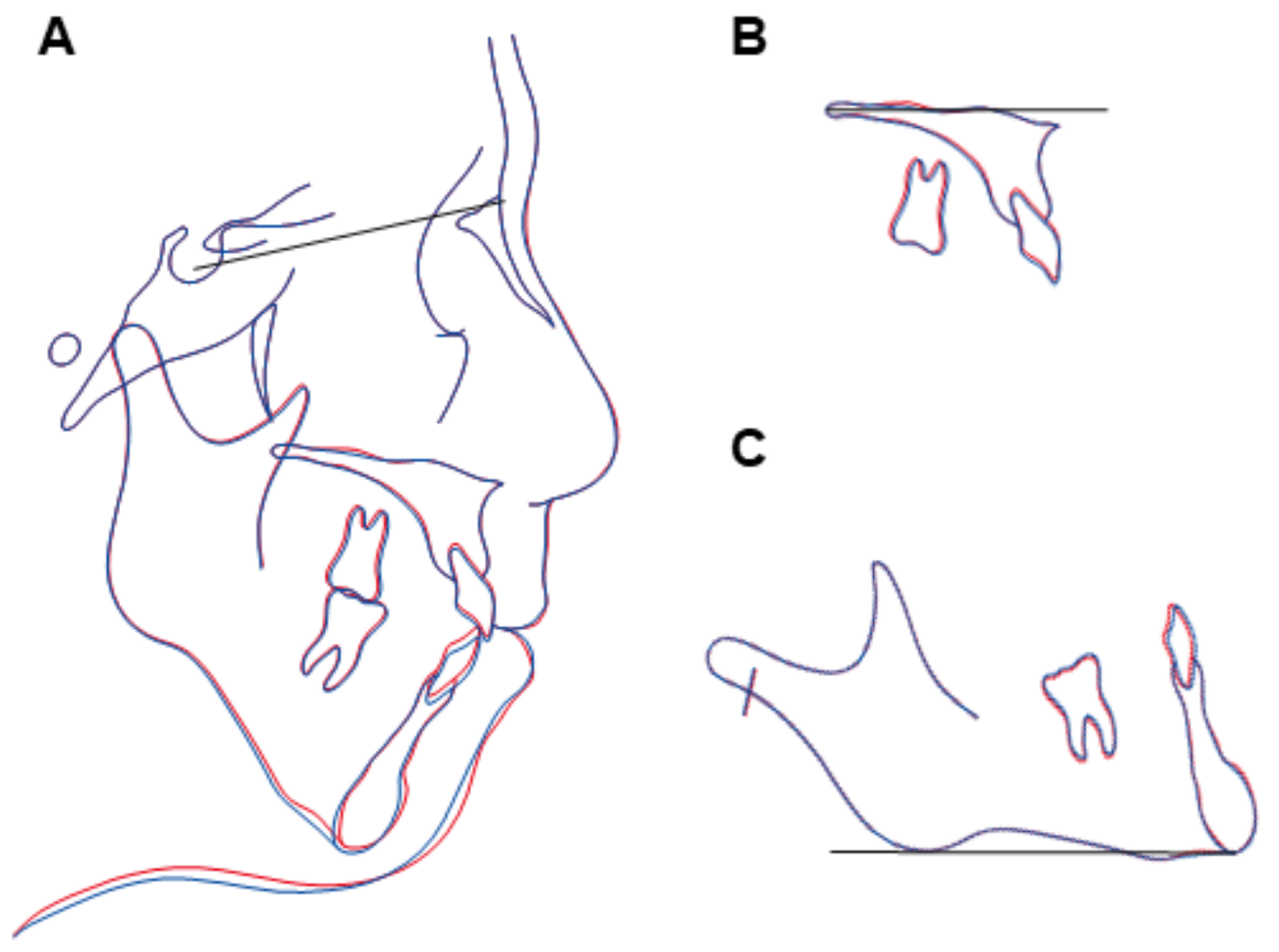

Figure 11

Superimposed cephalometric tracings show the changes from the post-treatment (red line) to postretention (blue line) stages. A, The overall superimposition on the sella-nasion plane at the sella. B, The superimposition on the initial palatal plane at the PNS. C, The superimposition on the mandibular plane at the menton. 